Homology, Homotopy and Applications, vol.16(1), 2014, pp.159-165

\title{
COMPLEXIFICATION AND HOMOTOPY
}

\author{
WOJCIECH KUCHARZ AND ŁUKASZ MACIEJEWSKI
}

(communicated by Claude Cibils)

\begin{abstract}
Let $Y$ be a real algebraic variety. We are interested in determining the supremum, $\beta(Y)$, of all nonnegative integers $n$ with the following property: For every $n$-dimensional compact connected nonsingular real algebraic variety $X$, every continuous map from $X$ into $Y$ is homotopic to a regular map. We give an upper bound for $\beta(Y)$, based on a construction involving complexification of real algebraic varieties. In some cases, we obtain the exact value of $\beta(Y)$.
\end{abstract}

\section{Introduction and main results}

In the present paper we continue the line of research undertaken in $[\mathbf{2}, \mathbf{5}]$. Our goal is to identify new obstructions to representing homotopy classes of continuous maps, between real algebraic varieties, by regular maps. We use the term real algebraic variety to mean a locally ringed space isomorphic to an algebraic subset of $\mathbb{R}^{N}$, for some $N$, endowed with the Zariski topology and the sheaf of real-valued regular functions (such an object is called an affine real algebraic variety in [1]). Morphisms between real algebraic varieties are called regular maps. Each real algebraic variety carries also the Euclidean topology, which is induced by the usual metric on $\mathbb{R}$. Unless explicitly stated otherwise, all topological notions relating to real algebraic varieties refer to the Euclidean topology.

In $[\mathbf{2}]$, a numerical invariant $\beta(Y)$ was defined for any real algebraic variety $Y$. Recall that $\beta(Y)$ is the supremum of all nonnegative integers $n$ with the following property: For every $n$-dimensional compact connected nonsingular real algebraic variety $X$, every continuous map from $X$ into $Y$ is homotopic to a regular map. The exact value of $\beta(Y)$ is known only in some special cases. The main result of [2] is an upper bound for $\beta(Y)$. For any nonnegative integer $k$, let $H_{\text {alg }}^{k}(Y ; \mathbb{Z} / 2)$ denote the subgroup consisting of all algebraic cohomology classes in the cohomology group $H^{k}(Y ; \mathbb{Z} / 2)$ (cf. [1] for $Y$ compact and nonsingular, and [2] for $Y$ arbitrary). According to $[\mathbf{2}$, Theorem 2.9], $\beta(Y) \leqslant k$ if $H_{\mathrm{alg}}^{k}(Y, \mathbb{Z} / 2) \neq 0$ for some $k \geqslant 1$.

In this paper we make use of a cohomology subgroup $H_{\mathbb{C}}^{k}(Y ; \mathbb{Q})$ of $H^{k}(Y ; \mathbb{Q})$, defined below, and prove that $\beta(Y) \leqslant k$ if $H_{\mathbb{C}}^{k}(Y ; \mathbb{Q}) \neq 0$ for some $k \geqslant 1$ (cf. Theorem 1.2). Furthermore, $\beta(Y)=0$ if $H_{\mathbb{C}}^{1}(Y ; \mathbb{Q}) \neq 0$ (cf. Theorem 1.3), whereas $\beta(Y)=$

The first author was partially supported by NCN grant 2011/01/B/BT1/01289.

Received October 24, 2013, revised January 14, 2014; published on April 22, 2014. 2010 Mathematics Subject Classification: 14P05, 14P25.

Key words and phrases: real algebraic variety, regular map, homotopy, complexification. Article available at http://dx.doi.org/10.4310/HHA.2014.v16.n1.a9

Copyright (C) 2014, International Press. Permission to copy for private use granted. 
$k-1$ if $Y$ is $(k-1)$-connected and $H_{\mathbb{C}}^{k}(Y ; \mathbb{Q}) \neq 0$ for some $k \geqslant 2$ (cf. Theorem 1.4).

Let $V$ be a compact nonsingular real algebraic variety. A nonsingular projective complexification of $V$ is a pair $(\mathbb{V}, e)$, where $\mathbb{V}$ is a nonsingular projective scheme over $\mathbb{R}$ and $e: V \rightarrow \mathbb{V}(\mathbb{C})$ is an injective map such that $\mathbb{V}(\mathbb{R})$ is Zariski dense in $\mathbb{V}$, $e(V)=\mathbb{V}(\mathbb{R})$, and $e$ induces a biregular isomorphism between $V$ and $\mathbb{V}(\mathbb{R})$. Here the set $\mathbb{V}(\mathbb{R})$ of real points of $\mathbb{V}$ is regarded as a subset of the set $\mathbb{V}(\mathbb{C})$ of complex points of $\mathbb{V}$. The existence of $(\mathbb{V}, e)$ follows from Hironaka's theorem on resolution of singularities [6] (cf. also [7] for a very readable exposition). If $\operatorname{dim} V \geqslant 2$, then $V$ admits infinitely many pairwise nonisomorphic projective complexifications, for $\mathbb{V}$ can be blown up along a nonsingular center disjoined form $\mathbb{V}(\mathbb{R})$. In view of this nonuniqueness, it is remarkable that for any commutative ring $R$ and any nonnegative integer $k$, the submodule

$$
H_{\mathbb{C}}^{k}(V ; R):=e^{*}\left(H^{k}(\mathbb{V}(\mathbb{C}) ; R)\right)
$$

of the cohomology $R$-module $H^{k}(V ; R)$, where

$$
e^{*}: H^{*}(\mathbb{V}(\mathbb{C}) ; R) \rightarrow H^{*}(V ; R)
$$

denotes the homomorphism induced by $e$, does not depend on the choice of $(\mathbb{V}, e)$. This is proved in [9] for $V$ orientable over $R$, and in [4] for arbitrary $V$. Note that in $[\mathbf{4}, \mathbf{9}]$ the authors use different notation for our $H_{\mathbb{C}}^{k}(-; R)$. As proved in $[\mathbf{4}, \mathbf{9}]$, the $R$-modules $H_{\mathbb{C}}^{k}(-; R)$ have the expected functorial property: If $h: V \rightarrow W$ is a regular map between compact nonsingular real algebraic varieties, then

$$
h^{*}\left(H_{\mathbb{C}}^{k}(W ; R)\right) \subseteq H_{\mathbb{C}}^{k}(V ; R) .
$$

The reader who wishes to find results comparing $H_{\mathbb{C}}^{k}(-; R)$ and $H^{k}(-; R)$ may consult $[8]$.

We extend the definition of $H_{\mathbb{C}}^{k}(-; R)$ as follows. For any real algebraic variety $X$, let $H_{\mathbb{C}}^{k}(X ; R)$ denote the set of all cohomology classes $u$ in $H^{k}(X ; R)$ of the form $u=\varphi^{*}(v)$, where $\varphi: X \rightarrow V$ is a regular map into a compact nonsingular real algebraic variety $V$ and $v$ is a cohomology class in $H_{\mathbb{C}}^{k}(V ; R)$.

Proposition 1.1. For any real algebraic variety $X$ and any nonnegative integer $k$, the set $H_{\mathbb{C}}^{k}(X ; R)$ forms a submodule of the cohomology $R$-module $H^{k}(X ; R)$. Furthermore, if $f: X \rightarrow Y$ is a regular map between real algebraic varieties, then

$$
f^{*}\left(H_{\mathbb{C}}^{k}(Y ; R)\right) \subseteq H_{\mathbb{C}}^{k}(X ; R)
$$

Proof. Let $\varphi_{i}: X \rightarrow V_{i}$ be a regular map into a compact nonsingular real algebraic variety $V_{i}$ for $i=1,2$. The regular map

$$
\left(\varphi_{1}, \varphi_{2}\right): X \rightarrow V_{1} \times V_{2}
$$

satisfies $\pi_{i} \circ\left(\varphi_{1}, \varphi_{2}\right)=\varphi_{i}$, where $\pi_{i}: V_{1} \times V_{2} \rightarrow V_{i}$ is the canonical projection. If $v_{i}$ is a cohomology class in $H^{k}\left(V_{i} ; R\right)$ for $i=1,2$, then

$$
\varphi_{1}^{*}\left(v_{1}\right)+\varphi_{2}^{*}\left(v_{2}\right)=\left(\varphi_{1}, \varphi_{2}\right)^{*}\left(\pi_{1}^{*}\left(v_{1}\right)+\pi_{2}^{*}\left(v_{2}\right)\right) .
$$

If $v_{i}$ is in $H_{\mathbb{C}}^{k}\left(V_{i} ; R\right)$ for $i=1,2$, then $\pi_{1}^{*}\left(v_{1}\right)+\pi_{2}^{*}\left(v_{2}\right)$ is in $H_{\mathbb{C}}^{k}\left(V_{1} \times V_{2} ; R\right)$. It follows that $\varphi_{1}^{*}\left(v_{1}\right)+\varphi_{2}^{*}\left(v_{2}\right)$ belongs to $H_{\mathbb{C}}^{k}(X ; R)$. Consequently, $H_{\mathbb{C}}^{k}(X ; R)$ is a submodule of the $R$-module $H^{k}(X ; R)$. 
Let $\psi: Y \rightarrow W$ be a regular map into a compact nonsingular real algebraic variety $W$. For any cohomology class $w$ in $H^{k}(W ; R)$,

$$
f^{*}\left(\psi^{*}(w)\right)=(\psi \circ f)^{*}(w) .
$$

Since $\psi \circ f$ is a regular map, it follows that $f^{*}\left(H_{\mathbb{C}}^{k}(Y ; R)\right) \subseteq H_{\mathbb{C}}^{k}(X ; R)$.

We now announce three results whose proofs will be given in Section 2.

Theorem 1.2. Let $Y$ be a real algebraic variety. If $H_{\mathbb{C}}^{k}(Y ; \mathbb{Q}) \neq 0$ for some positive integer $k$, then $\beta(Y) \leqslant k$.

In some cases, we get stronger results.

Theorem 1.3. Let $Y$ be a real algebraic variety. If $H_{\mathbb{C}}^{1}(Y, \mathbb{Q}) \neq 0$, then $\beta(Y)=0$.

We also have a criterion for the equality $\beta(Y)=k-1$, where $k \geqslant 2$.

Theorem 1.4. Let $Y$ be a real algebraic variety. Assume that $Y$ is $(k-1)$-connected for some integer $k \geqslant 2$. If $H_{\mathbb{C}}^{k}(Y ; \mathbb{Q}) \neq 0$, then $\beta(Y)=k-1$.

In some cases, our results are stronger than those that can be deduced from $[\mathbf{2}, \mathbf{5}]$.

Example 1.5. For any positive integer $k$, the real algebraic variety

$$
\Sigma^{k}=\left\{\left(x_{0}, \ldots, x_{k}\right) \in \mathbb{R}^{k+1} \mid x_{0}^{4}+\cdots+x_{k}^{4}=1\right\}
$$

is nonsingular and diffeomorphic to the unit $k$-sphere. By [8, Example 2.3], we have

$$
H_{\mathbb{C}}^{k}\left(\Sigma^{k} ; \mathbb{Q}\right)=H^{k}(\Sigma ; \mathbb{Q})
$$

and hence Theorems 1.3 and 1.4 imply the equality $\beta\left(\Sigma^{k}\right)=k-1$. Since the real curve $\Sigma^{1}$ is not rational, one easily obtains $\beta\left(\Sigma^{1}\right)=0$ without referring to Theorem 1.3; cf. [2, Example 1.7(v)]. On the other hand, for $k \geqslant 2$, the methods developed in $[\mathbf{2}, \mathbf{5}]$ give only the inequalities $k-1 \leqslant \beta\left(\Sigma^{k}\right) \leqslant k$.

\section{Proofs}

For any $k$-dimensional compact oriented smooth (of class $\mathcal{C}^{\infty}$ ) manifold $K$, let $[K]$ denote its fundamental class in $H_{k}(K ; \mathbb{Z})$. If $K$ is a subspace of a topological space $P$, we denote by $[K]_{P}$ the homology class in $H_{k}(P ; \mathbb{Z})$ represented by $K$, that is, $[K]_{P}=i_{*}([K])$, where $i: K \hookrightarrow P$ is the inclusion map.

As usual, for any nonnegative integer $d$, we denote by $\mathbb{S}^{d}$ the unit $d$-sphere,

$$
\mathbb{S}^{d}=\left\{\left(u_{0}, \ldots, u_{d}\right) \in \mathbb{R}^{d+1} \mid u_{0}^{2}+\cdots+u_{d}^{2}=1\right\} .
$$

The following refinement of Thom's representability theorem [11, Théorème III.4] will play a key role.

Theorem 2.1. Let $Y$ be a topological space that is homotopically equivalent to a $C W$ complex, $k$ a positive integer, and $\alpha$ a homology class in $H_{k}(Y ; \mathbb{Z})$. Then there exist a $k$-dimensional compact oriented stably parallelizable smooth manifold $K$, a continuous map $f: K \rightarrow Y$, and a positive integer $c$ such that $f_{*}([K])=c \alpha$. Furthermore, if $\alpha$ is represented by a singular cycle with support contained in a connected component of $Y$, then the manifold $K$ can be chosen connected. 
Proof. We may assume without loss of generality that $Y$ is a compact and connected CW-complex that is embedded in $\mathbb{R}^{p}$ for some $p \geqslant 2 k+2$. The argument used in $\left[\mathbf{1 1}\right.$, pp. 57, 58] implies the existence of a retraction $Q \rightarrow Y$, where $Q \subseteq \mathbb{R}^{p}$ is a $p$ dimensional compact connected smooth submanifold with boundary, containing $Y$ in its interior. Let $P$ be the double of $Q$. By construction, $P$ is a compact connected parallelizable smooth manifold and there exists a retraction $r: P \rightarrow Y$. Choose an orientation of $P$. Let $i: Y \hookrightarrow P$ be the inclusion map. Let $d=p-k$ and let $u$ be the cohomology class in $H^{d}(P ; \mathbb{Z})$ that corresponds via the Poincare duality to the homology class $i_{*}(\alpha)$ in $H_{k}(P ; \mathbb{Z})$. Since $p \leqslant 2 d-2$, according to Serre's result [10, p. 289, Proposition $\left.2^{\prime}\right]$ we can find a continuous map $\varphi: P \rightarrow \mathbb{S}^{d}$ and a positive integer $c$ such that

$$
\varphi^{*}\left(s_{d}\right)=c u
$$

where $s_{d}$ is a generator of the cohomology group $H^{d}\left(\mathbb{S}^{d} ; \mathbb{Z}\right) \cong \mathbb{Z}$. We can assume that the map $\varphi$ is smooth. By Sard's theorem, there exists a regular value $y$ in $\mathbb{S}^{d}$ of the map $\varphi$. If the smooth submanifold $L:=\varphi^{-1}(y)$ of $P$ is suitably oriented, then

$$
[L]_{P}=c i_{*}(\alpha) .
$$

Obviously, the normal bundle of $L$ in $P$ is trivial. Since $\operatorname{dim} L=k \geqslant 1$, we can perform the connected sum operation on the connected components of $L$. This can be done inside $P$ since $k \leqslant p-2$ and hence $P \backslash L$ is connected. In other words, we join, in a suitable way, the connected components of $L$ with $k$-dimensional tubes in $P$. Thus we obtain a compact connected oriented smooth submanifold $K$ of $P$, which is homologous to $L$ and whose normal bundle in $P$ is trivial. Note that

$$
[K]_{P}=c i_{*}(\alpha) \text {. }
$$

If $j: K \hookrightarrow P$ is the inclusion map and $f:=r \circ j: K \rightarrow Y$, then $j_{*}([K])=[K]_{P}=$ $c i_{*}(\alpha)$ and

$$
f_{*}([K])=r_{*}\left(j_{*}([K])\right)=c r_{*}\left(i_{*}(\alpha)\right)=c(r \circ i)_{*}(\alpha)=c \alpha .
$$

It remains to show that the smooth manifold $K$ is stably parallelizable. For any smooth manifold $M$, let $\tau_{M}$ denote its tangent bundle. We have $\tau_{K} \oplus \nu=\left.\tau_{P}\right|_{K}$, where $\nu$ is the normal bundle of $K$ in $P$. Hence $K$ is stably parallelizable, the vector bundles $\nu$ and $\tau_{P}$ being trivial.

Let $S$ be a topological space. For any cohomology class $u$ in $H^{k}(S ; \mathbb{Q})$ and any homology class $\alpha$ in $H_{k}(S ; \mathbb{Z})$, we denote by $\langle u, \alpha\rangle$ their Kronecker index. If $u \neq 0$, then we can choose $\alpha$ so that $\langle u, \alpha\rangle \neq 0$.

Recall that any real algebraic variety is homotopically equivalent to a compact polyhedron (thus, homotopically equivalent to a compact CW-complex); cf. [1, Theorem 9.2.1, Corollary 9.3.7].

Proof of Theorem 1.2. Assume that $H_{\mathbb{C}}^{k}(Y ; \mathbb{Q}) \neq 0$, where $k \geqslant 1$. Let $u$ be a nonzero cohomology class in $H_{\mathbb{C}}^{k}(Y ; \mathbb{Q})$. Choose a homology class $\alpha$ in $H_{k}(Y ; \mathbb{Z})$ satisfying

$$
\langle u, \alpha\rangle \neq 0
$$

and such that it is represented by a singular cycle with support contained in a connected component of $Y$. According to Theorem 2.1, there exist a $k$-dimensional compact connected oriented smooth manifold $K$, a continuous map $f: K \rightarrow Y$, and a 
positive integer $c$ such that

$$
f_{*}([K])=c \alpha
$$

and $K$ is stably parallelizable. By [8, Corollary 2.9], there exists a nonsingular real algebraic variety $X$ diffeomorphic to $K \times \mathbb{S}^{1}$ and satisfying

$$
H_{\mathbb{C}}^{k}(X ; \mathbb{Q})=0 .
$$

Let $\varphi: X \rightarrow K \times \mathbb{S}^{1}$ be a smooth diffeomorphism and let $\pi: K \times \mathbb{S}^{1} \rightarrow K$ be the canonical projection. It suffices to prove that the continuous map

$$
g:=f \circ \pi \circ \varphi: X \rightarrow Y
$$

is not homotopic to a regular map. This can be done as follows. Let $z_{0}$ be a point in $\mathbb{S}^{1}$ and $K_{0}:=\varphi^{-1}\left(K \times\left\{z_{0}\right\}\right)$. Then

$$
g_{*}\left(\left[K_{0}\right]_{X}\right)=f_{*}\left(\pi_{*}\left(\left[K \times\left\{z_{0}\right\}\right]_{K \times \mathbb{S}^{1}}\right)\right)=f_{*}([K])=c \alpha .
$$

Consequently,

$$
\left\langle g^{*}(u),\left[K_{0}\right]_{X}\right\rangle=\left\langle u, g_{*}\left(\left[K_{0}\right]_{X}\right)\right\rangle=c\langle u, \alpha\rangle \neq 0,
$$

which implies $g^{*}(u) \neq 0$. In view of Proposition 1.1 and the equality $H_{\mathbb{C}}^{k}(X ; \mathbb{Q})=0$, we would have $g^{*}(u)=0$ if $g$ were homotopic to a regular map. The proof is complete.

The following fact will be useful.

Example 2.2. If $\mathbb{T}^{n}=\mathbb{S}^{1} \times \cdots \times \mathbb{S}^{1}$ is the $n$-fold product, then

$$
H_{\mathbb{C}}^{k}\left(\mathbb{T}^{n} ; \mathbb{Q}\right)=0 \quad \text { for every } k \geqslant 1
$$

Indeed, the real projective line $\mathbb{P}^{1}$ (regarded as a scheme over $\mathbb{R}$ ) is a nonsingular projective complexification of $\mathbb{S}^{1}$, and hence the $n$-fold product $\mathbb{V}=\mathbb{P}^{1} \times \mathbb{R} \cdots \times \mathbb{R} \mathbb{P}^{1}$ is a nonsingular projective complexification of $\mathbb{T}^{n}$. Let

$$
e: \mathbb{V}(\mathbb{R})=\mathbb{P}^{1}(\mathbb{R}) \times \cdots \times \mathbb{P}^{1}(\mathbb{R}) \hookrightarrow \mathbb{V}(\mathbb{C})=\mathbb{P}^{1}(\mathbb{C}) \times \cdots \times \mathbb{P}^{1}(\mathbb{C})
$$

be the inclusion map. It suffices to note that $e^{*}\left(H^{k}(\mathbb{V}(\mathbb{C}) ; \mathbb{Q})\right)=0$ for every $k \geqslant 1$. This follows from the Künneth formula in cohomology since $\mathbb{P}^{1}(\mathbb{R})$ is homeomorphic to $\mathbb{S}^{1}$ while $\mathbb{P}^{1}(\mathbb{C})$ is homeomorphic to $\mathbb{S}^{2}$.

Henceforth, for each nonnegative integer $d$, we choose an orientation of $\mathbb{S}^{d}$ and regard $\mathbb{S}^{d}$ as an oriented manifold.

Lemma 2.3. Let $Y$ be a real algebraic variety, $k$ a positive integer, and $u$ a cohomology class in $H_{\mathbb{C}}^{k}(Y ; \mathbb{Q})$. Assume that there exists a continuous map $f: \mathbb{S}^{k} \rightarrow Y$ such that $\left\langle u, f_{*}\left(\left[\mathbb{S}^{k}\right]\right)\right\rangle \neq 0$. Then $\beta(Y) \leqslant k-1$.

Proof. Let $\mathbb{T}^{k}=\mathbb{S}^{1} \times \cdots \times \mathbb{S}^{1}$ be the $k$-fold product. We endow $\mathbb{T}^{k}$ with an orientation and choose a continuous map $\varphi: \mathbb{T}^{k} \rightarrow \mathbb{S}^{k}$ satisfying

$$
\varphi_{*}\left(\left[\mathbb{T}^{k}\right]\right)=\left[\mathbb{S}^{k}\right] .
$$

It suffices to prove that the continuous map

$$
g:=f \circ \varphi: \mathbb{T}^{k} \rightarrow Y
$$

is not homotopic to a regular map. In view of Proposition 1.1 and Example 2.2, we 
would have $g^{*}(u)=0$ if $g$ were homotopic to a regular map. However

$$
\left\langle g^{*}(u),\left[\mathbb{T}^{k}\right]\right\rangle=\left\langle u, g_{*}\left(\left[\mathbb{T}^{k}\right]\right)\right\rangle=\left\langle u, f_{*}\left(\left[\mathbb{S}^{k}\right]\right)\right\rangle \neq 0,
$$

which implies $g^{*}(u) \neq 0$.

Proof of Theorem 1.3. Assume that $H_{\mathbb{C}}^{1}(Y ; \mathbb{Q}) \neq 0$, and let $u$ be a nonzero cohomology class in $H_{\mathbb{C}}^{1}(Y ; \mathbb{Q})$. We can find a continuous map $f: \mathbb{S}^{1} \rightarrow Y$ for which $\left\langle u, f_{*}\left(\left[\mathbb{S}^{1}\right]\right)\right\rangle \neq$ 0 . This assertion holds since the homology classes of the form $f_{*}\left(\left[\mathbb{S}^{1}\right]\right)$ generate the group $H_{1}(Y ; \mathbb{Z})$. In order to complete the proof it suffices to apply Lemma 2.3 with $k=1$.

Proof of Theorem 1.4. Assume that $H_{\mathbb{C}}^{k}(Y ; \mathbb{Q}) \neq 0$, and let $u$ be a nonzero cohomology class in $H_{\mathbb{C}}^{k}(Y ; \mathbb{Q})$. Since $Y$ is $(k-1)$-connected, according to the Hurewicz theorem, each homology class in $H_{k}(Y ; \mathbb{Z})$ is of the form $h_{*}\left(\left[\mathbb{S}^{k}\right]\right)$ for some continuous map $h: \mathbb{S}^{k} \rightarrow Y$. Hence there exists a continuous map $f: \mathbb{S}^{k} \rightarrow Y$ such that $\left\langle u, f_{*}\left(\left[\mathbb{S}^{k}\right]\right)\right\rangle \neq$ 0 . It follows from Lemma 2.3 that $\beta(Y) \leqslant k-1$. We get the equality $\beta(Y)=k-1$, since, for every compact polyhedron $X$ of dimension at most $k-1$, every continuous map from $X$ into $Y$ is null homotopic; cf. [3, p. 509, Corollary 13.14].

\section{References}

[1] J. Bochnak, M. Coste, M.-F. Roy, Real Algebraic Geometry, vol. 36, Ergebnisse der Mathematik und ihrer Grenzgebiete, Berlin, Springer, 1998.

[2] J. Bochnak and W. Kucharz, Real algebraic morphisms represent few homotopy classes, Math. Ann. 337 (2007), 909-921.

[3] G. E. Bredon, Topology and geometry, Springer-Verlag, 1993.

[4] S. Dolega, Complexification and cohomology in real algebraic geometry, Ph.D. Thesis, University of New Mexico, 2004.

[5] R. Gilhoni, Second order homological obstructions in real algebraic manifolds, Topology Appl. 154 (2007), 3090-3094.

[6] H. Hironaka, Resolution of singularities of an algebraic variety over a field of characteristic zero, Ann. of Math. 79 (1964), 109-326.

[7] J. Kollár, Lectures on Resolution of Singularities, Ann. of Math. Studies, vol. 166, Princeton University Press, Princeton, NJ, 2007.

[8] W. Kucharz and K. Kurdyka, Complexification of algebraic models of smooth manifolds, J. London Math. Soc. 84 (2011), 325-343.

[9] Y. Ozan, On homology of real algebraic varieties, Proc. Amer. Math. Soc. 129 (2001), 3167-3175.

[10] J.-P. Serre, Groupes d'homotopie et classes des groupes abeliens, Ann. of Math. 58 (1953), 258-294.

[11] R. Thom, Quelques propriétés globales des variétés différentiables, Comment, Math. Helv. 28 (1954), 17-86. 
Wojciech Kucharz Wojciech.Kucharz@im.uj.edu.pl

Department of Mathematics, Faculty of Mathematics and Computer Science, Jagiellonian University, Łojasiewicza 6, 30-348 Kraków, Poland

Łukasz Maciejewski Lukasz.Maciejewski@im.uj.edu.pl

Department of Mathematics, Faculty of Mathematics and Computer Science, Jagiellonian University, Łojasiewicza 6, 30-348 Kraków, Poland 